\title{
Total Knee Arthroplasty for Osteoarthritis in a Patient with Nail Patella Syndrome - A Case Report
}

\author{
Antonio Filipe Serrano (iD) \\ Pedro Manuel Serrano (D) ${ }^{2}$ \\ 'Medical Department - USF Lidador, \\ ACeS Grande Porto III Maia/Valongo, \\ Maia, Portugal; ${ }^{2}$ Orthopedic Department \\ - Centro Hospitalar Universitário do \\ Porto, Porto, Portugal
}

\begin{abstract}
Hereditary osteo-onychodysplasia (or nail patella syndrome) is a rare autosomal dominant disease defined by an association of severe characteristics, with a classic clinical tetrad of dysplasia of the fingernails, patellar hypoplasia or absence, presence of iliac horns, and deformities of the elbow, although renal, ocular and neurological involvement may also be present. The main orthopedic complaint of these patients is patellar instability associated with pain and functional limitation. Most of the literature regarding the treatment of NPS-related patellar problems, concerns pediatric population and there is no treatment algorithm defined for adult patients. NPS has a wide range of presentations in skeletally mature patients, and its severity is also very variable. The general practitioner should be familiar with NPS's clinical presentation spectrum, and the orthopedic surgeon with treatment options, in order to provide the most adequate treatment for each patient. Although there is no cure for NPS, several treatments have been useful in the symptomatic treatment of changes in the knees. We describe a rare clinical case of a $47-$ year-old female patient with NPS who underwent total knee arthroplasty for knee osteoarthritis.
\end{abstract}

Keywords: hereditary onycho-osteodysplasia, patellar aplasia, total knee arthroplasty, adult

\section{Introduction}

Nail patella syndrome (NPS), also known as hereditary onycho-osteodysplasia or Fong disease, is rare ( 1 in 50,000, but may be higher because of undiagnosed individuals with a mild phenotype) autosomal dominant disease defined by the association of severe characteristics, with a classic clinical presentation that includes dysplasia of the fingernails, hypoplasia or complete absence of the patella, iliac horns and deformities of the elbow. Renal, ocular, and neurological symptoms may also be present.

Physical and radiological examination is key in diagnosing NPS, although genetic testing and renal biopsy may be useful for confirmation. ${ }^{1}$

Clinical manifestations are variable, both in severity and frequency. ${ }^{2}$ For instance, a severe aspect of NPS may affect an individual that has milder or no manifestations elsewhere. Although diagnosis at birth is possible, it is not uncommon to find families that remain undiagnosed for several years or even generations. ${ }^{3}$

The earliest description of patients suffering from this disease was published in the scientific literature in the year $1820 .^{4}$ 
Most of the literature regarding the treatment of NPSrelated patellar problems, concerns pediatric population, ${ }^{5}$ and there is no defined treatment algorithm for adult patients. ${ }^{6}$

The main orthopedic complaint of these patients is patellar instability associated with pain and functional limitation.

The purpose of this work is to describe and discuss the clinical case of an NPS patient that underwent Total knee arthroplasty (TKA) because of knee osteoarthritis. We present this case not only for the rarity of this syndrome but especially for the selected treatment option and its very positive outcome.

\section{Case Report}

A 47-year-old Caucasian woman was admitted in Centro Hospitalar Universitário do Porto's Orthopedic
Department via her general practitioner (GP) with an history of NPS and osteochondritis dissecans of the external femoral condyle of the left knee (underwent surgery in 1989 at another hospital). After referral to our hospital for knee pain and a sensation of blockage of the knee, because of knee osteoarthritis, a detailed study was performed. This study included conventional and extra-long $\mathrm{x}$-rays of the lower extremities (Figure 1), Computed Tomography (CT)-Scan to measure the angle between the interepicondylar line of the femur and the posterior condylar line (Figure 2) and a three-dimensional CT reconstruction of the femur and tibia (Figure 3). These studies helped assess bone morphology and evaluate the rotational changes of limb.
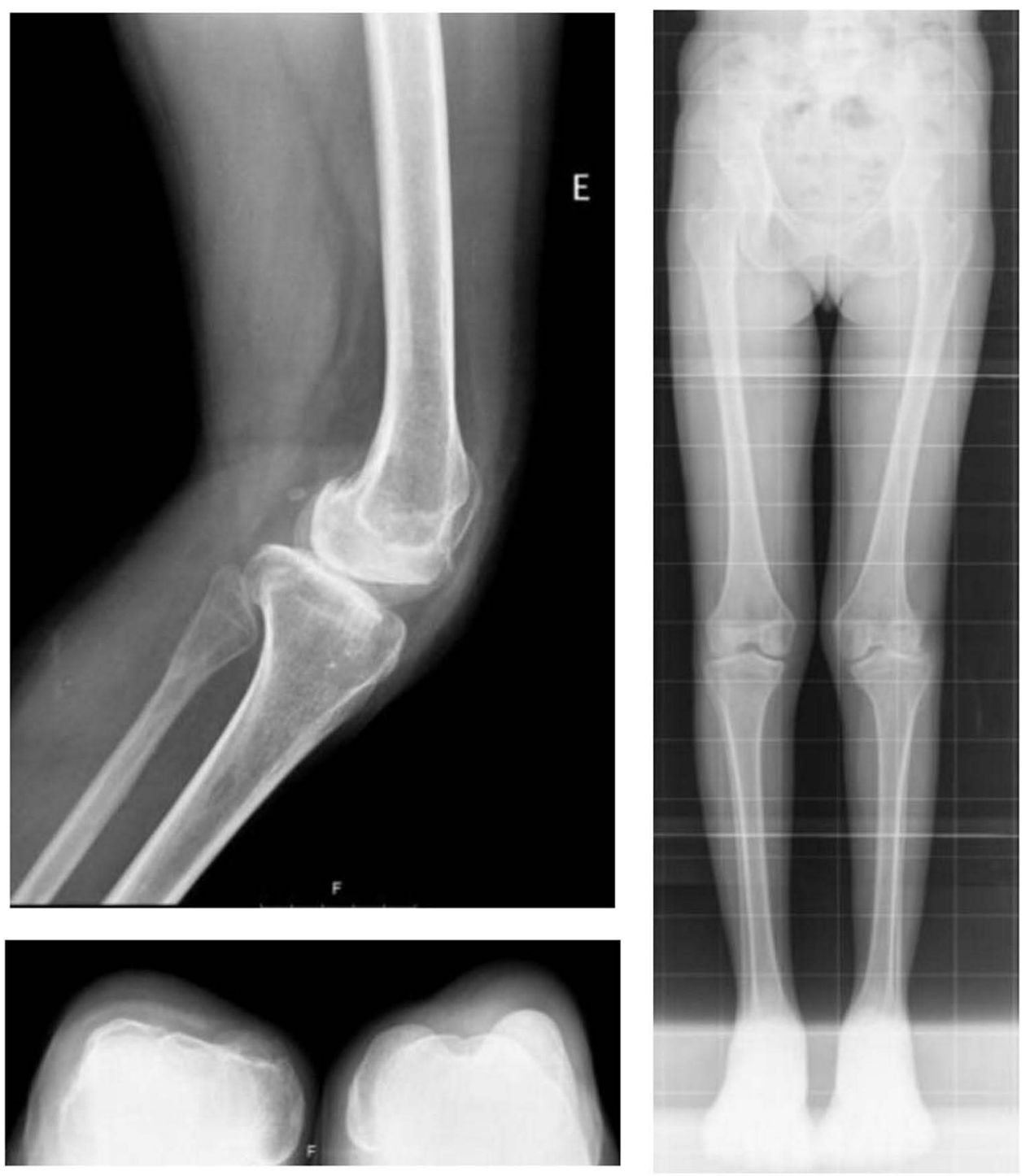

Figure I Pre-operatory X-rays; long-leg (left), side view (top right), axial view (bottom right). 


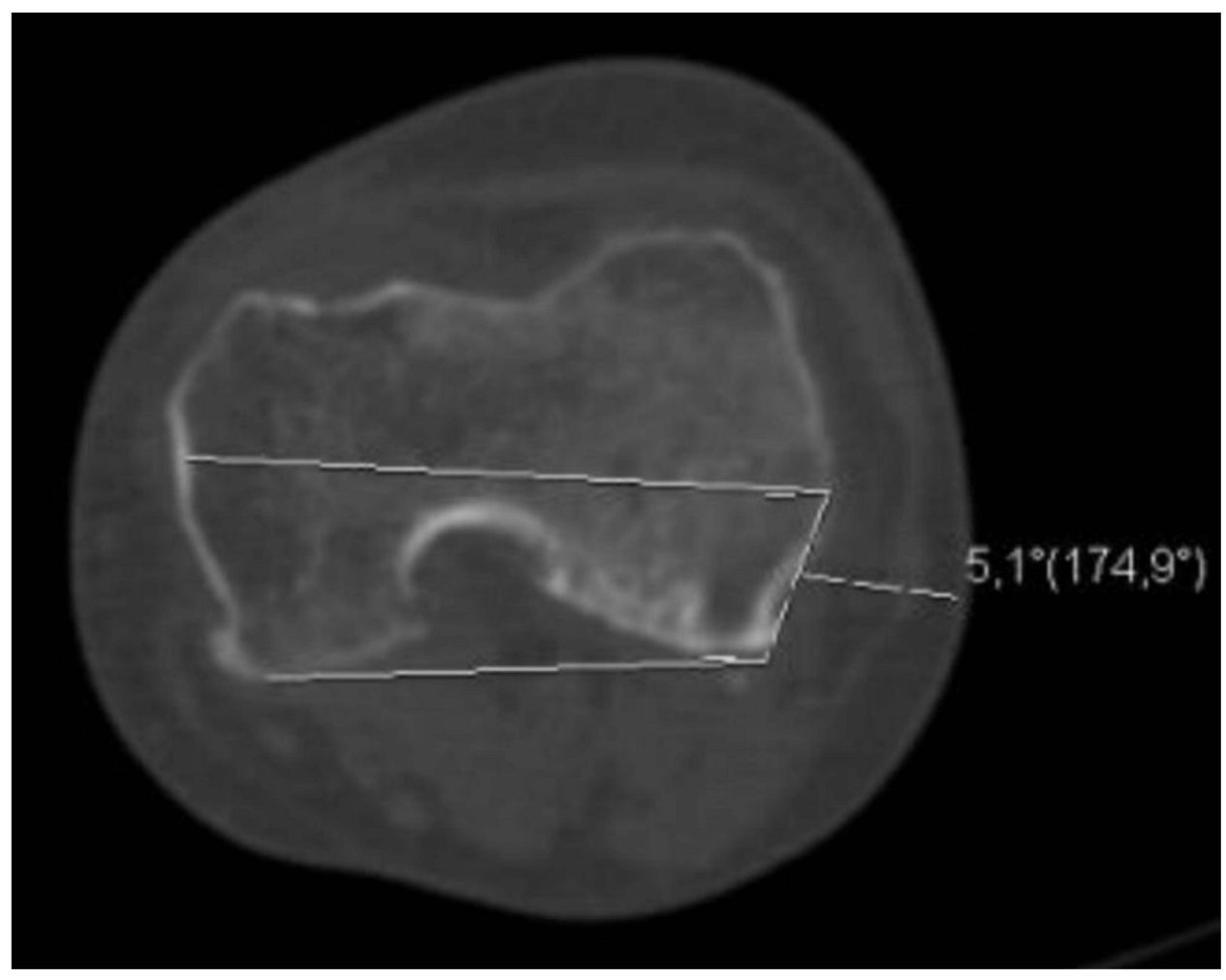

Figure 2 Pre-operatory CT-scan (measuring the angle between the interepicondylar line of the femur and the posterior condylar line).

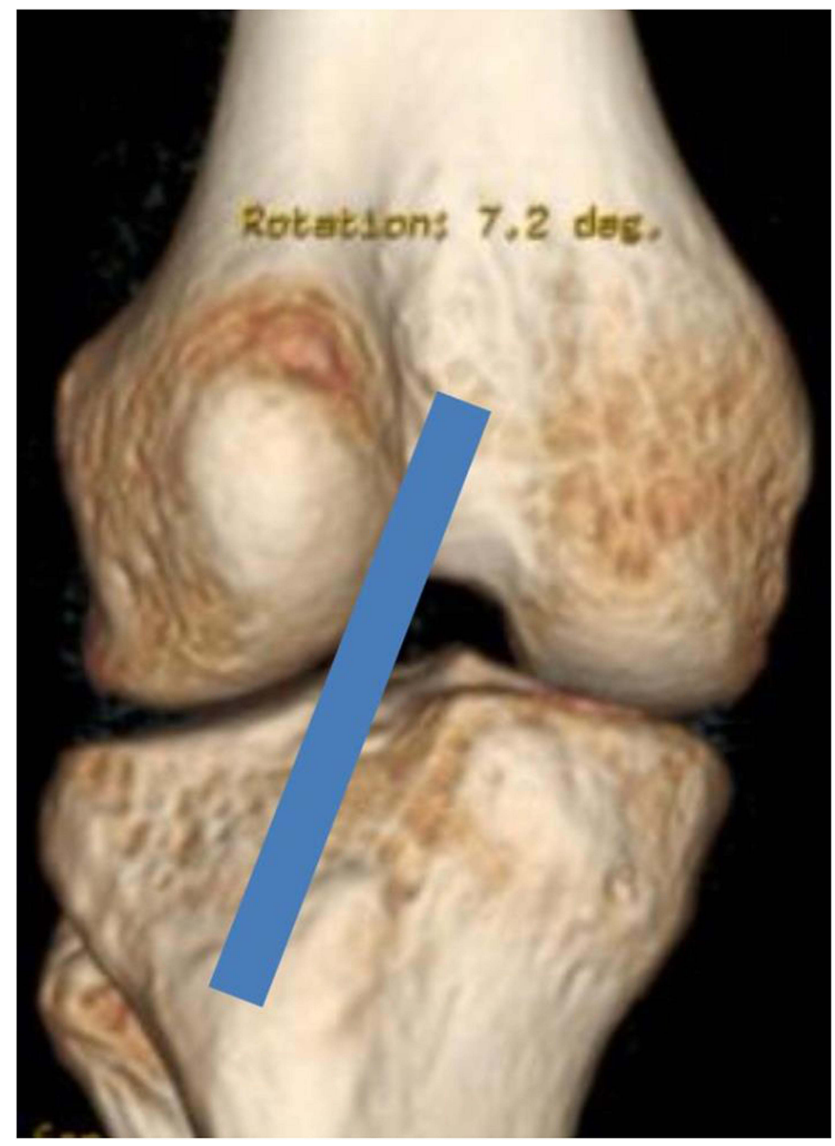

Figure 3 Pre-operatory 3 dimensional reconstruction of the knee joint showing a lateral translation of the anterior tibial tubercle. 

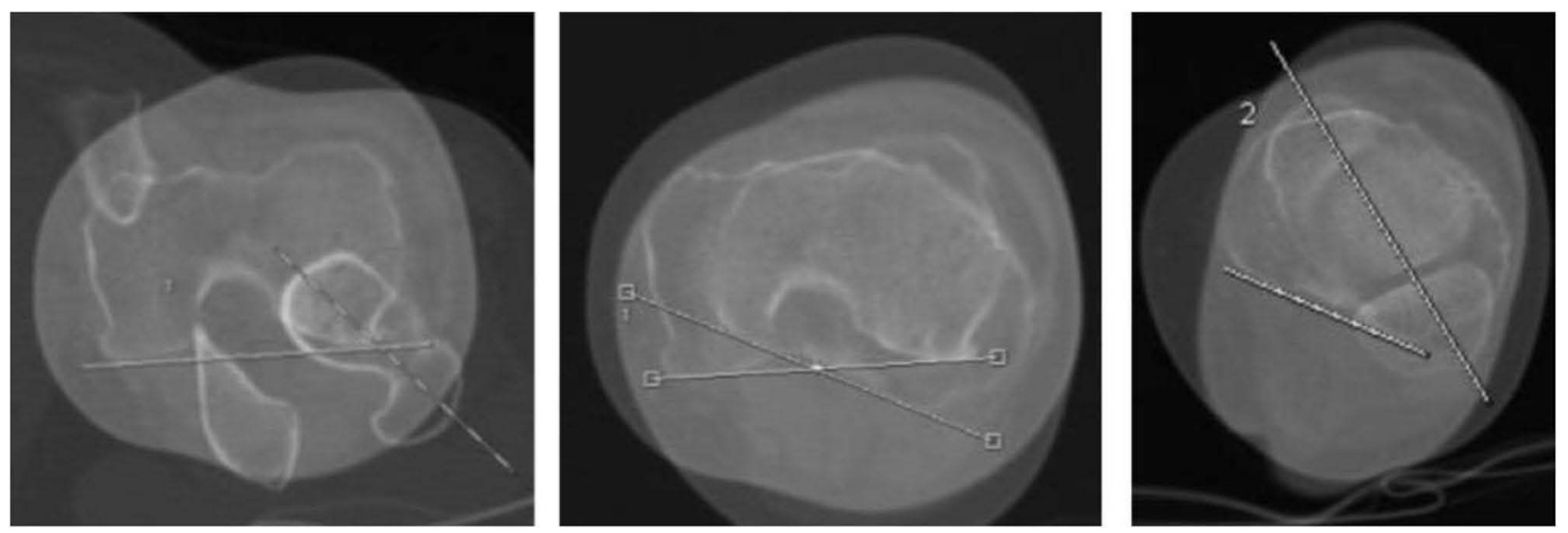

Figure 4 Pre-operatory CT-scan revealing significant rotation malalignment.

X-rays (Figure 1) revealed a valgus alignment of the left limb, knee osteoarthritis (Kellgren- Lawrence IV) essentially of the external condyle, patellar aplasia, and the CT-scan also detected an important rotation malalignment (Figure 4) with 47 degrees of femoral anteversion, femorotibial rotation of 23 degrees and an external tibial torsion of 36 degrees.

Treatment options were discussed with the patient as well as their expected risks and benefits.

Total knee arthroplasty was performed with a standard NEXGEN prosthesis in February 2014. The surgery and postoperative period underwent without any complications. The patient started early mobilization with partial load. Radiographic exam 8 weeks after surgery was normal (Figure 5). Physiotherapy program was maintained for the next 2 months and the ability to walk without external support and without claudication was achieved at the 10th week postop. The patient was very satisfied with the clinical outcome and reported a significant increase in quality of life, especially because of the ability to use stairs, sit, stand up and walk for longer periods without pain. Objectively, the patient presented a full range of passive mobility and a slight limitation in active knee flexion at the 3 years post-operative follow-up consultation.

The Knee Injury and Osteoarthritis Outcome Score (KOOS) was used to compare the knee pain at the preoperatory stage and at 3 years follow-up. Results were graded from 0 to 100 (Table 1).

\section{Conclusion}

NPS has a wide range of presentations in skeletally mature patients, and its severity is also very variable. The general practitioner (GP) should be familiar with NPS's clinical presentation spectrum, and the orthopedic surgeon with treatment options, in order to provide the most adequate treatment for each patient.

Although there is no cure for NPS, several options have been useful in treating its symptoms. Some authors recommend a conservative attitude towards cases of congenital dislocation of the patella, which can pass through analgesia, physiotherapy and immobilization. A patellectomy is indicated in cases with unfavorable evolution after conservative treatment, patellofemoral arthrosis, pain and significant functional limitation. Total knee arthroplasty, although rarely described in the literature for treating these patients, appears to be indicated in selected cases, where there is severe osteoarthritis, even if the patella is absent, as in the case here described.

We present this case not only for the rarity of this syndrome but especially for the selected treatment option and its very positive outcome.

The treatment was effective, and the patient improved not only regarding quality of life but also pain and joint mobility. The authors wish to highlight the importance of a multidisciplinary approach to the patient where communication between the GP, orthopedics, radiology and physiotherapy is needed. The patient's commitment, motivation and involvement 

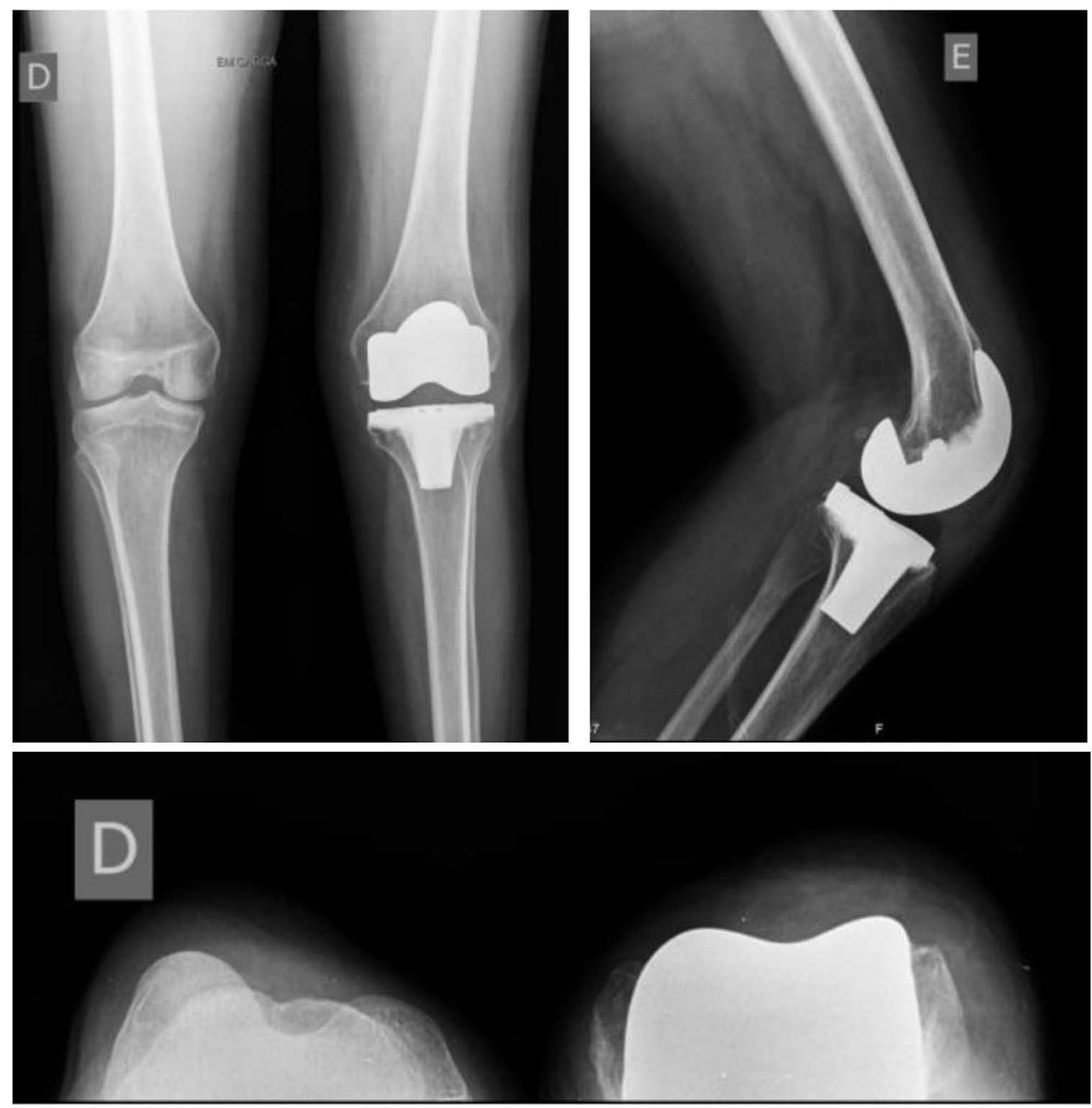

Figure 5 Post-operatory X-rays; front view (top left), side view (top right), axial view (bottom right).

during the whole process was also crucial. In our opinion, these factors combined with an uneventful surgery positively influenced the final outcome.

Table I Knee Injury and Osteoarthritis Outcome Score

\begin{tabular}{|l|c|c|}
\hline & Pre-Op & Post-Op \\
\hline Pain & 36 & 28 \\
\hline Symptoms & 43 & 21 \\
\hline Daily living & 21 & 26 \\
\hline Sports/Recreation & 15 & 0 \\
\hline Quality of life & 31 & 19 \\
\hline
\end{tabular}

Note: Knee pain compared between the pre-operatory stage and the last follow-up (3 years after surgery).

Abbreviations: Pre-op, preoperative; Post-op, postoperative.

\section{Statement of Informed Consent}

The patient was informed and agreed that data concerning the case would be submitted for publication. The institutional review board also confirmed patient's consent, confirmed that data was anonymized and agreed with publication.

\section{Disclosure}

The authors report no conflicts of interest in this work.

\section{References}

1. Lovelace PD, May LA. Nail patella syndrome. In: StatPearls [Internet]. Treasure Island (FL): StatPearls Publishing; January, 2021 [updated June 5, 2021]. Available from: https://www.ncbi. nlm.nih.gov/books/NBK559190/. Accessed December 20, 2021.

2. Turner JW. An hereditary arthrodysplasia associated with hereditary dystrophy of the nails. JAMA. 1933;100(12):882-884. doi:10.1001/ jama.1933.02740120020008 
3. Lucas GL, Opitz JM. The nail-patella syndrome. Clinical and genetical aspects of 5 kindreds with 38 affected family members. J Pediatr. 1966;68:273-288. doi:10.1016/S0022-3476(66)80159-2

4. Duncan JG, Souter WA. Hereditary onycho-osteodysplasia. $J$ Bone Joint Surg. 1963;45B(2):242-258. doi:10.1302/0301-620X.45B2.242

5. Tognetti L, Baldassarri M, Fava F, et al. Novel mutation in LMX1B gene in a newborn with nail-patella syndrome: clinical and dermoscopic findings. Pediatr Dermatol. 2020;37(6):1205-1206. doi:10.1111/ pde. 14337
6. Louboutin L, Wascher D, Neyret P. Management of patellar problems in skeletally mature patients with nail-patella syndrome. Knee Surg Sports Traumatol Arthrosc. 2017;25:3012-3016. doi:10.1007/ s00167-016-4044-y

\section{Publish your work in this journal}

The International Medical Case Reports Journal is an international, peer-reviewed open-access journal publishing original case reports from all medical specialties. Previously unpublished medical posters are also accepted relating to any area of clinical or preclinical science. Submissions should not normally exceed 2,000 words or 4 published pages including figures, diagrams and references. The manuscript management system is completely online and includes a very quick and fair peer-review system, which is all easy to use. Visit http://www.dovepress.com/testimonials.php to read real quotes from published authors. 